\title{
A Model of a Multi-Site Fishery with Variable Price: from Over-Exploitation to Sustainable Fisheries
}

\author{
S. Ly ${ }^{1}$, F. Mansal ${ }^{1}$, M. Baldé ${ }^{1}$, T. Nguyen-Huu ${ }^{2}$, P. Auger $^{1,3}$ \\ ${ }^{1}$ Université Cheikh-Anta-Diop, Dakar, Sénégal \\ 2 IRD UMI IMMISCO, 32 av. Henri Varagnat, 93140 Bondy cedex, France \\ 3 IXXI, ENS Lyon, 15 parvis René Descartes, BP 7000, 69342 Lyon Cedex 07
}

\begin{abstract}
We present a mathematical model of a fishery on several sites with a variable price. The model takes into account the evolution during the time of the resource, fishes and boats movements between the different sites, fishing effort and price that varies with respect to supply and demand. We suppose that boats and fishes movements as well as prices variations occur at a fast time scale. We use methods of aggregation of variables in order to reduce the number of variables and we derive a reduced model governing two global variables, respectively the biomass of the resource and the fishing effort of the whole fishery. We look for the existence of equilibria of the aggregated model. We show that the aggregated model can have 1, 2 or 3 non trivial equilibria. We show that a variation of the total number of sites can induce a switch from over-exploitation to sustainable fisheries.
\end{abstract}

Keywords and phrases: dynamical systems, multi-site fishery, variable price, demand function, equilibrium, stability, optimum capture

Mathematics Subject Classification: 92D25, 91B276, 34A33

\section{Introduction}

Mathematical modelling of renewable resources has received a lot of attention since many years. The originality of bio-economic models is to take into account biological aspects as well as economic ones. We refer to both classical and recent books $[7,10]$. In most mathematical models, (usually a set of coupled ODEs), a first equation describes the time variation of the resource, commonly logistically growing and harvested according to a Schaefer function [25]. A key concept in Bio-economics relates to the maximum sustainable yield (MSY) which is defined as the largest catch that can be taken from a fishery stock over an indefinite period. Moreover, a second equation is sometimes added in order to describe the time variation of the fishing effort or more generally (in case of another resource than fish), the number of firms (assumed to be homogeneous) involved in the exploitation of the resource at different time [6,26,27]. Many mathematical fishery models consider a constant market price of the resource. However, some authors assumed that the price can depend on some variables such as the resource density, or the fishing effort [6]. In a previous contribution [5], a third equation was even added in order to take into account the time variation of the resource as a result of demand and supply.

*Corresponding author. E-mail: tri.nguyen-huu@ird.fr

(C) EDP Sciences, 2013 
There was also a lot of interest in modeling multi-site fisheries. Indeed, environment heterogeneity influences spatial interactions in fishery activities. The fishery is thus considered as a network of patches connected by migration [24]. The fish population is sometimes represented as a metapopulation in the sea [18] focusing in local extinction / recolonization processes at different scales. Patches can correspond to different fishing areas as well as Marine Protected Areas (MPAs), artificial habitats/reefs. In particular, we focus here on artificial sites called Fish Aggregating Devices (FADs) or Artificial Habitats (AHs) $[16,19]$. FADs are permanent or temporary structures floating at the surface or at midwater, and are used to attract fishes [9]. The reason why fishes aggregate around those devices is still not clearly established. Nowadays, the use of FADs is extensive, particularly the use of large-scale industrial FADs focused on capturing large schools of tuna [17,29], but often without any regulation [11]. More than half of the world's tropical tuna catches are being made around FADs [12]. Tuna or similar big pelagic fishes move fast and are attracted by FADS [13], where they stay around for a relatively short time, a few days $[8,23]$ and return to the open sea as a free stock. Moreover, tuna fishes frequently leave a given FAD before going to another one [8] or returning to the same one.

Understanding FADs effects on fish stock, catch and economy is a key aspect for improving fisheries management and addressing legal issues related to the way they are used. We present a bioeconomic model of a fishery that explicitly considers effects of FADs. The aim of this contribution is to present a mathematical bio-economic model which describes the time variation of the fish stock and the fishing effort by coupling the two previous aspects, i.e. price variation of the resource according to demand and supply, and heterogeneous environment, represented as a set of patches connected by migrations. Our approach particularly focuses on the effect of the number of patches (FADs) on the dynamics of the fishery and the switch between an over-exploitation fishery to a sustainable one. We show that changing adequatly the number of sites allows to modify fish stock and fishing effort in order to eventually switch from over-exploitation to a sustainable fishery while increasing the total capture.

Our work consists of three sections. In section 2, we build a fishery model: the main point is to consider a model with an extra equation for the variation of the market price. We assume that the adjustment of the market price occurs rapidly with respect to fish growth, investment (new boats entering the fishery) and capture. This allows us to consider a slow-fast model in which two time scales are involved. In section 3, we take advantage of these two time scales to reduce the dimension of the complete model and derive a global model at the slow time scale by use of aggregation of variables methods $[2-4,14,15]$. In section 4, we study the properties of this approximate model which governs the total fish density and the total fishing fleet. In this section, we study the total capture and discuss the relationship between the number of sites and total catch.

\section{A multisite fishery model}

Here we study a model of a multi-site fishery that is structured in different sites/patches that are associated with different FADs. In our model, fishing vessels are assumed to capture fishes only around a given FAD. There is no fishing in the open sea. As a consequence the fish stock is divided into two main components, a free stock in the open sea and an harvested stock that stays around the system of FADs. Therefore, the model must take into account fishes movement from the open sea to the FADs as well as vessels movements from FAD to FAD where they catch fishes, [22]. Similarly, there is evidence that fishing vessels also move frequently from FAD to FAD in order to capture fishes. It is therefore reasonable to assume that there exist two time scales, a fast time scale corresponding to fishes and vessels movements and a slow one in comparison, corresponding to fish population growth, capture and fishing effort evolution.

We consider the coast as a linear chain of $L$ artificial sites (either FADs or AHs), with fishes moving between the sites and a free stock, unattached to the sites. We build a model governing fish densities and fishing efforts. Let $n_{s}(t)$ be the fish density of the free stock at time $t$, let $n_{i}(t)$ be the fish density on site $i$ at time $t$, and similarly, let $E_{i}(t)$ be the fishing effort on site $i$ at time $t, i \in[1, L]$. In the free stock 
and the different sites, fish population follows a logistic growth. We consider that fishes have potentially different growth rates on the free stock and on the sites, which we call $r_{s}$ and $r_{1}$, respectively.

We assume that the system has a global and constant fish carrying capacity $K$. We suppose that a constant proportion $0<\alpha<1$ of the stock is free, whereas the remaining proportion is attached to the sites. We call $k_{s}$ and $k_{i}, i \in[1, L]$, the carrying capacity of the free stock and site $i$, respectively, with

$$
\begin{gathered}
k_{s}=\alpha K \\
\sum_{i=1}^{L} k_{i}=(1-\alpha) K
\end{gathered}
$$

On site $i$, fishing effort varies proportionnally to the difference between profit $\left(p q n_{i}\right.$, where $p$ is the price per fish unit and $q$ the fishing efficiency) and fixed maintenance $\operatorname{costs} c$.

We assume that fishes movements and boats displacements occur at a fast time scale $\tau$, whereas fish growth and the dynamics of the fishery occur at a slow time scale $t=\varepsilon \tau$, with $\varepsilon \ll 1$ being a small dimensionless parameter. Fishes move between every site and the free stock, whereas boats move between neighbouring sites. It makes sense to assume that the movement rate for boats from a site to a neighbouring site depends on the distance between these sites. Therefore, we assume symmetric movement rates for boats: $\beta_{i+1, i}=\beta_{i, i+1} ; i \in\{1, \ldots, L-1\}$. We also assume that fishes movement rates are inversely proportional to the carrying capacity of the site (or the free stock). The movement rate between free stock and site $i$ (resp. site $i$ and free stock) is denoted $m_{i s}$ (resp. $m_{s i}$ ) and reads:

$$
m_{i s}=\frac{m_{0}}{k_{s}} ; \quad m_{s i}=\frac{m_{0}}{k_{i}} \quad \text { for } i \in\{1, \ldots, L\}
$$

where $m_{0}$ is a positive constant that represents the migration rate when the carrying capacity of the departure site is set to 1 . Thus, according to eq. (2.3), fish are more likely to remain on sites offering large carrying capacities, i.e., on sites where the resources are abundant.

We also include in the model the dynamics of fish price. In several works, the price of the resource is constant or a function of fish capture $[6,26,27]$. At our knowledge, little attention has been paid to model the fishery dynamics as a dynamical system in which the market price of the resource is also a variable. However, the validity of the assumption of a constant fish price seems limited to small scale fisheries. Furthermore, according to classical economic theory [28], the price variation depends on the gap between a demand function (that is the quantity of fish purchased by consumers) and the supply which is no more than the catch. This suggests to consider that the price is not a constant but a variable. As a result, price is not constant in the present model and depends on the variables such as the resource or the fishing effort. We suppose that the price of the capture varies at fast time scale and depends on a demand function $D(p)$ and a supply which is simply given by the instantaneous capture on all sites.

$$
\frac{d p}{d \tau}=\Phi\left(D(p)-q \sum_{i=1}^{L} n_{i} E_{i}\right)
$$

$\Phi$ is a positive constant and $q$ is the catchability assumed homogeneous.

The following system describes the time evolution of the fishery: 


$$
\left\{\begin{aligned}
\frac{d n_{s}}{d \tau} & =\sum_{i=1}^{L} m_{s i} n_{i}-\sum_{i=1}^{L} m_{i s} n_{s}+\varepsilon r_{s} n_{s}\left(1-\frac{n_{s}}{k_{s}}\right) \\
\frac{d n_{i}}{d \tau} & =m_{i s} n_{s}-m_{s i} n_{i}+\varepsilon\left(r_{1} n_{i}\left(1-\frac{n_{i}}{k_{i}}\right)-q n_{i} E_{i}\right) \\
\frac{d E_{i}}{d \tau} & =\beta_{i, i-1} E_{i-1}+\beta_{i, i+1} E_{i+1}-\left(\beta_{i-1, i}+\beta_{i+1, i}\right) E_{i}+\varepsilon\left(-c+p q n_{i}\right) E_{i} \\
\frac{d p}{d \tau} & =\Phi\left(D(p)-q \sum_{i=1}^{L} n_{i} E_{i}\right)
\end{aligned}\right.
$$

Later on, we will consider a linear demand function such as in [20]. Such a linear function is assumed to be a decreasing function of market price with a maximum value $A$ when the price is equal to zero:

$$
D(p)=A-\gamma p
$$

where $\gamma$ is a positive constant.

\section{Aggregated model and analysis}

In order to analyse the dynamics of the model, we apply aggregation methods as described in [3] to obtain a reduced system: a two dimensional system of ordinary differential equations governing the total fish stock and the total fishing effort at the slow time scale. The sufficient conditions for a system to be perfectly as well as approximately aggregated have been investigated in the frame of general population models in $[14,15,21]$. In our case, the aggregation of the complete model consists in supposing that the fast dynamics has attained a stable equilibrium and in substituting this fast equilibrium into the equations of the complete model.

In [3], solutions of complete and aggregated models have been compared in an example of a predatorprey ODE model in a patchy environment. it was shown that aggregation methods can be successfully applied (i.e. both solutions remain very close of each other) when parameter epsilon is smaller than 0.1 , which corresponds to one order of magnitude between the two time scales. In our fishery model, assuming that reproduction as well as variation of number of boats of the fishing fleet occur annually, considering fishes and boats movements at a time scale corresponding to the day or the month is sufficient to use aggregation methods.

\subsection{Aggregated model}

We now determine the fast equilibrium. The fast dynamics corresponds to fishes and boats dispersal from FAD to FAD, dispersal between FADs and free stock, and price variation. We assume that fishes and boats move at a fast time scale in comparison to fish growth, i.e. the variation of stocks due to dispersal is greater than variation due to demography. We first analyse the fast system by assuming that $\varepsilon=0$ in eq. (2.5).

This model is similar to the one presented in [5] in which the effect of the number of sites was studied but with a constant price, contrary to the present case. At the fast time scale, the total fish density $n(t)=\sum_{i=1}^{L} n_{i}(t)+n_{s}(t)$ remains constant. It is easy to check that there is a unique positive and stable fast equilibrium for fish densities (see Appendix 1):

$$
\begin{gathered}
n_{s}^{*}=\frac{k_{s}}{K} n=\alpha n \\
n_{i}^{*}=\frac{k_{i}}{K} n \quad \text { with } \quad \sum_{n=1}^{L} n_{i}^{*}=(1-\alpha) n
\end{gathered}
$$


The previous equations predict that the equilibrium proportion of fish in a site is proportional to the site carrying capacity. Similarly, we set $\varepsilon=0$ into the equation of the fishing efforts. At the fast time scale, the total fishing effort $E(t)=\sum_{i=1}^{L} E_{i}(t)$ also remains constant, and there exists a unique positive and stable equilibrium for fishing efforts (see Appendix 1):

$$
E_{i}^{*}=\frac{E}{L}
$$

Considering $D(p)=A-\gamma p$ where $A$ and $\gamma$ are the maximum price and a positive constant respectively, price at fast equilibrium is given by:

$$
p^{*}=\frac{A-Q n E}{\gamma}
$$

where $Q=q(1-\alpha) / L$ is the global catchability parameter.

The aggregated model is obtained by substituting the fast and stable equilibrium for price (eq 3.4), fishes (eqs. 3.1 and 3.2) and boats (eq. 3.3) movement into the complete system (eq. 2.5) and by adding the $L+1$ fishes and the $L$ boats equations. This leads to the following structurally stable model (see Appendix 2):

$$
\left\{\begin{array}{l}
\frac{d n}{d t}=r n\left(1-\frac{n}{K}\right)-Q n E \\
\frac{d E}{d t}=\left(-c+\frac{A-Q n E}{\gamma} Q n\right) E
\end{array}\right.
$$

Where $r=\alpha r_{s}+(1-\alpha) r_{1}$ is the global growth rate.

\subsection{Equilibria and local stability analysis}

The $n$-nullclines are: $n=0$ and $E=\frac{r}{Q}\left(1-\frac{n}{K}\right)$ and $E$-nullclines are: $E=0$ and $E=\frac{\gamma}{Q n}\left(\frac{A}{\gamma}-\frac{c}{Q n}\right)$. We obtain the equilibria $(K ; 0),(0 ; 0)$ and possibly interior points $\left(n^{*} ; E^{*}\right)$ which are solutions of:

$$
\left\{\begin{array}{l}
E=\frac{r}{Q}\left(1-\frac{n}{K}\right) \\
E=\frac{\gamma}{Q n}\left(\frac{A}{\gamma}-\frac{c}{Q n}\right)
\end{array}\right.
$$

Solution for system (3.6) is obtained by solving a third degree equation in variable $n$. There can be one to three equilibria in the positive quadrant that we note $\left(\bar{n}_{1} ; \bar{E}_{1}\right),\left(\bar{n}_{2} ; \bar{E}_{2}\right)$ and $\left(\bar{n}_{3} ; \bar{E}_{3}\right)$ (or sometimes $(\bar{n} ; \bar{E})$ in a generic way). We now perform local stability analysis for those equilibria. We determine the Jacobian matrix for the different equilibria for system (3.5) and determine stability from the signs of its determinant and trace :

$-E_{0}=(0 ; 0)$ :

$$
J(0 ; 0)=\left[\begin{array}{cc}
r & 0 \\
0 & -c
\end{array}\right]
$$

$\operatorname{det} J(0 ; 0)=-r c$, hence the equilibrium point $(0 ; 0)$ is always a saddle point.

$-E_{K}=(K ; 0)$

$$
J(K ; 0)=\left[\begin{array}{cc}
-r & -Q K \\
0 & -c+\frac{A Q K}{\gamma}
\end{array}\right]
$$

$\operatorname{det} J(K ; 0)=-r\left(-c+\frac{A Q K}{\gamma}\right)$ and $\operatorname{Tr} J(K ; 0)=-r-c+\frac{A Q K}{\gamma}$. If $K<\frac{c \gamma}{A Q}$ then $(K ; 0)$ is a stable equilibrium, otherwise it is a saddle point. 
- Non-trivial equilibria: we denote those equilibria $(\bar{n}, \bar{E})$ in a general way. The jacobian matrix reads

$$
J(\bar{n} ; \bar{E})=\left[\begin{array}{cc}
-\frac{r}{K} \bar{n} & -Q \bar{n} \\
\frac{Q \bar{E}}{\gamma}(A-2 Q \bar{n} \bar{E}) & -\frac{Q^{2} \bar{n}^{2} \bar{E}}{\gamma}
\end{array}\right]
$$

The trace and the determinant of the Jacobian matrix at the equilibrium point $(\bar{n} ; \bar{E})$ are respectively given by:

$$
\begin{aligned}
& \operatorname{Tr} J(\bar{n} ; \bar{E})=-\frac{r}{K} \bar{n}-\frac{Q^{2} \bar{n}^{2} \bar{E}}{\gamma}<0 \\
& \operatorname{det} J(\bar{n} ; \bar{E})=\frac{Q^{2} \bar{n} \bar{E}}{\gamma}\left(\frac{3 r}{K} \bar{n}^{2}-2 \bar{n} r+A\right)
\end{aligned}
$$

The sign of $\operatorname{det} J\left(n^{*} ; E^{*}\right)$ is the same than the sign of $\Psi(\bar{n})$, where $\Psi(\bar{n})=\frac{3 r}{K} \bar{n}^{2}-2 \bar{n} r+A$. The complete study of existence and stability is studied in Appendix 3 and can be summarized in two cases:

Case 1: $r<\frac{3 A}{K}$. The sign of $\Psi$ does not change and is always positive, and then $\operatorname{det} J(\bar{n} ; \bar{E})>0$. There are two subcases:

(a) $\frac{A}{\gamma} Q K<c$ : in this case, there is no positive equilibrium. $(K, 0)$ is then a stable equilibrium. This can be interpretated as the fact that it is not possible to have a profitable fishery: with a fish stock at carrying capacity and with maximum price $(A / \gamma)$, the income $\frac{A}{\gamma} Q K$ is inferior to maintenance costs c.

(b) $\frac{A}{\gamma} Q K>c$ : in this case, there is one stable equilibrium point in the positive quadrant. $(K ; 0)$ is then a saddle point.

Case 2: If $r>\frac{3 A}{K}$ there can be one stable equilibrium or three equilibria $\left(\bar{n}_{1} ; \bar{E}_{1}\right),\left(\bar{n}_{2} ; \bar{E}_{2}\right)$ and $\left(\bar{n}_{3} ; \bar{E}_{3}\right)$ in the positive quadrant. In this last case, the middle equilibrium $\left(\bar{n}_{2} ; \bar{E}_{2}\right)$ is a saddle while the two others are stable. This case is illustrated in Figure 1.

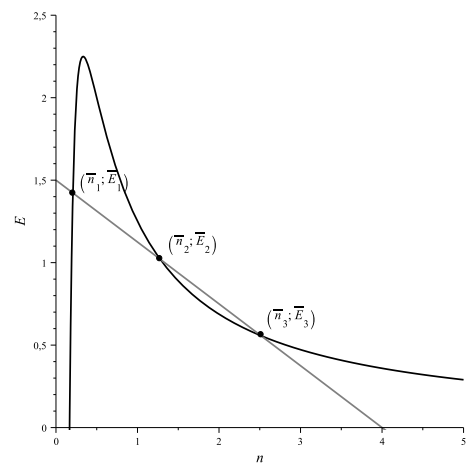

$(a)$

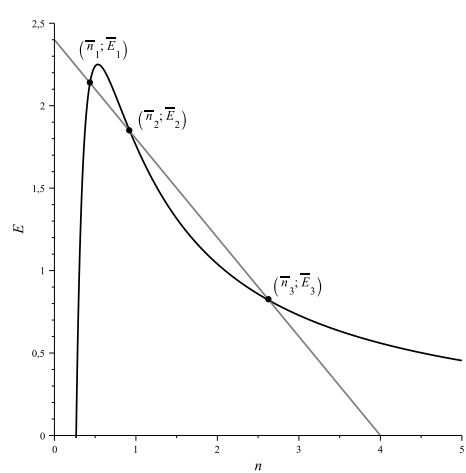

(b)

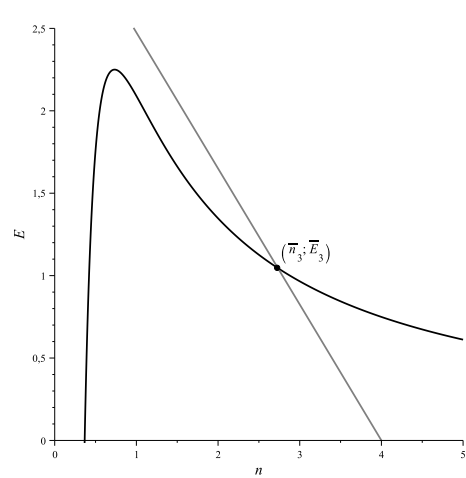

(c)

Figure 1. Equilibria $\left(\bar{n}_{i}, \bar{E}_{i}\right)$ for different numbers of FADs $L:(a) L=5,(b) L=8$ and (c) $L=11$. The equilibria are given by the intersections of the two curves given in eq. 3.6, represented in black and grey. Parameters value are $r=3, A=3, q=11.11, c=1$, $K=4, \gamma=1$ and $\alpha=0.1$. 


\section{Model discussion}

In this section, we discuss the results and focus on capture analysis. The total capture is given by:

$$
\bar{Y}=Q \bar{n} \bar{E}
$$

Values of $\bar{n}_{i}$ and $\bar{E}_{i}$ are solutions of third degree equations derived from system (3.6). They are represented graphically on Figure 1. The values of captures at equilibria are represented on Figure 2, and depend on $L$. Figure 1 shows equilibria in the phase plane $(n, E)$ for three different values of $L$. From Figures 1 and 2 , we determine that for a low number of sites, there can be two stable equilibria: $\left(\bar{n}_{1}, \bar{E}_{1}\right)$ and $\left(\bar{n}_{3}, \bar{E}_{3}\right)$ which are respectively over-exploitation equilibrium and sustainable equilibrium. Equilibrium $\left(\bar{n}_{1}, \bar{E}_{1}\right)$ corresponds to a over-exploited fishery, with a low fish stock, a high fishing effort and a low capture. On the contrary, equilibrium $\left(\bar{n}_{3}, \bar{E}_{3}\right)$ corresponds to a low fishing effort, resulting in a high fish stock, and in the end, a high total capture. The first equilibrium may be seen as an industrial exploitation while the last equilibrium would correspond to an artisanal local fishery or to a sustainable development fishery. Equilibrium $\left(\bar{n}_{2}, \bar{E}_{2}\right)$ is a saddle point, so there exists a separatrix which seperates the space $(n, E)$ in two domains in which the trajectories will tend toward the same equilibrium.

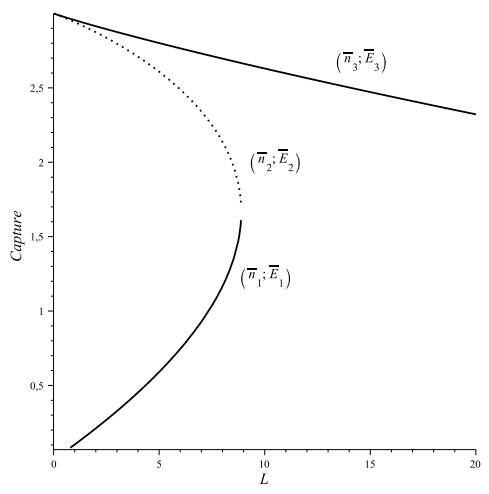

FIGURE 2. total capture corresponding respectively to equilibria $\left(\bar{n}_{1} ; \bar{E}_{1}\right),\left(\bar{n}_{2} ; \bar{E}_{2}\right)$ and $\left(\bar{n}_{3} ; \bar{E}_{3}\right)$, as a function of the number of sites. Solid lines represent stable equilibria $\left(\left(\bar{n}_{1} ; \bar{E}_{1}\right)\right.$ and $\left.\left(\bar{n}_{3}, \bar{E}_{3}\right)\right)$, while the dotted line represents the unstable equilibrium $\left(\bar{n}_{2} ; \bar{E}_{2}\right)$. Parameters value are $r=3, A=3, q=11.11, c=1, K=4, \gamma=1$ and $\alpha=$ 0.1. Parameter $L$ is supposed to be an integer. However, the curve are represented as continuous values for a better understanding of their variations.

Figure 2 shows that when the number of sites $L$ increases, the total capture at over-exploitation equilibrium increases. In the same time, total capture at sustainable equilibrium decreases. This appears also on Figure 1: when $L$ increases, the slope of the straight line increases; $\left(\bar{n}_{1} ; \bar{E}_{1}\right)$ and $\left(\bar{n}_{2} ; \bar{E}_{2}\right)$ eventually merge around the maximum of the black curve. When $L$ goes past a threshold $L_{t}$ (around 9 with the chosen set of parameters), equilibria $\left(\bar{n}_{1} ; \bar{E}_{1}\right)$ and $\left(\bar{n}_{2} ; \bar{E}_{2}\right)$ disappear, corresponding to a saddle-node bifurcation.

When fisheries present characteristics of over-exploitation, we deduce from the mathematical system that it is not possible to change to a sustainable fishery naturally. In the mathematical model, a major perturbation of the system is needed in order to make a trajectory "cross" the separatrix, and then enter the basin of attraction of the other equilibrium. However, one could wish to reach a sustainable state as it ensures a higher total catch. The behaviour described in this section can be turned as an advantage in order to switch from an over-exploitation equilibrium to a sustainable equilibrium. One should increase the number of sites over the critical threshold $L_{t}$. The total capture will first increase, then the overexploitation equilibrium will disappear, leaving the sustainable equilibrium as the only stable equilibrium 
in the positive quadrant. When the sustainable equilibrium is reached, decreasing slowly $L$ will increase the total capture while keeping the system at the sustainable equilibrium, even when the over-exploitation equilibrium reappears. Figure 3 illustrates this behaviour: between $t=0$ and $t=15, L=5$ and the system tends toward the over-exploitation equilibrium. Between $t=15$ and $t=25$, the number of FADs $L$ is increased to 11 . The system tends toward the sustainable equilibrium, which is at this time the only positive equilibrium. After $t=25$, the number of FADs is reduced to 5 . The system still tends toward the sustainable equilibrium. at this time, the over-exploitation equilibrium has reappeared, but cannot be reached from the current state. At the end of the simulation, the number of FADs is the same than at the begining, but the system has switched from the basin of attraction of over-exploitation equilibrium to the one of the sustainable equilibrium.

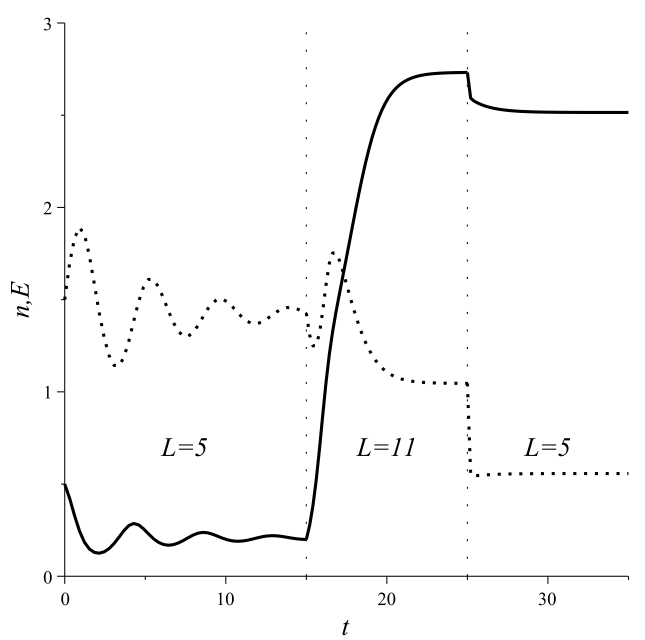

(a)

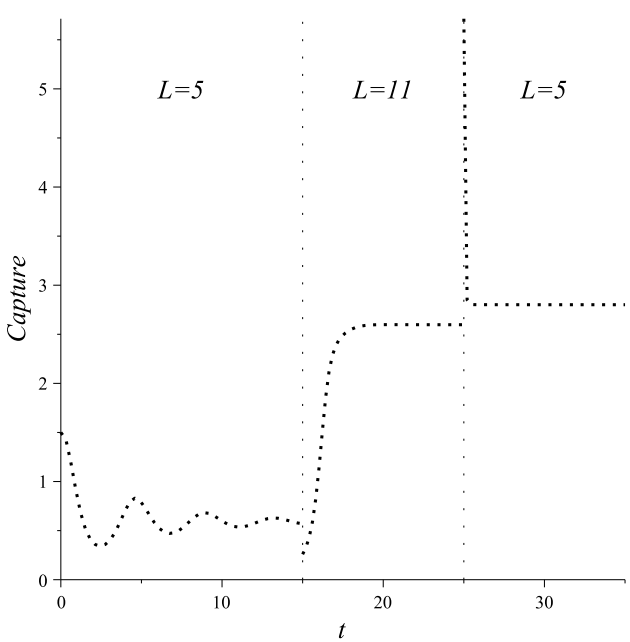

(b)

Figure 3. Dynamics obtained by modifying the number of FADs: (a) fish stock $n$ (solid line) and fishing effort $E$ (dotted line), (b) total capture. Between $t=0$ and $t=15$, $L=5$, between $t=15$ and $t=25, L=11$; for $t>25, L=5$.

\section{Conclusion and perspectives}

The model presented in this work includes two aspects of fisheries that are often neglected: the use of floating aggregation devices and economical aspects of the dynamics, relying on a variable price that depends on demand and supply. Aggregation of variables allowed to demonstrate that there exists an over-exploitation equilibrium, as well as a sustainable equilibrium, and that it was possible to define a strategy based on FADs number to switch from a state corresponding to over-exploitation to a sustainable one. Presently, most fisheries using FADs are over-exploited, and FADs are deployed in large amounts without regards to any regulation, see [11]. This corresponds to a state of the model that is close to the over-exploitation state equilibrium, $\left(n_{1}^{*} ; E_{1}^{*}\right)$. In many fisheries, more and more FADs are installed. The model presented here suggests that when the number of FADs becomes greater than a threshold corresponding to parameter $L_{t}$, the system is going to switch from the over-exploitation fishery to the sustainable one and it will remain in the sustainable state even when $L$ continues to increase. Such a result suggests that if research institutes or governmental organizations in charge of the management of a FAD fishery wish to maintain the fishery in a sustainable state, they should recommend firstly to increase the number of FADs to switch from an over-exploitation fishery to a sustainable one. They can then 
suppress several FADs progressively in order to remain in the sustainable equilibrium and to increase the total catch (see figure 2). Reaching sustainable exploitation leads to higher fish stocks which will provide a higher economic profit but which will protect fish stocks from extinction due to random demographic events or wrong evaluation of fish stocks and fishing efforts [7].

As perspectives, it will be very interesting to apply these results to real cases, like "Thiof" fisheries. This fish is the most appreciated in Senegal and as king of fishes. There are presently signs of overexploitation and new regulations including FADs deployments are needed. It may be interesting to regulate Thiof fishing by modifying the number of FADs, as it would easier to control than fishing activities themselves. We plan to make developments of the model to make it more specific to this case. The specific model should include a fine description of the environment geography (coastline...) as well as socioeconomics reality (detailed movement of boats according to fishing habits and port locations, workforce availability and management...).

The present model has been written has a general framework, without any specific behaviour for fishes or boats. In the present model, it has been assumed that boats movement where symmetrical, which is true when boats movement rates are influenced by the distance between two sites. In reality, boats movements may be also influenced by several factors such as the density of fishes on a given location but also port location, meteorology, ocean currents. Similarly, fish movements may also depend on geography, ocean currents, tide, etc. As a consequence, in order to extend the results to a specific case, one should carefully determine if assumptions made in this model still hold.

\section{Appendix}

\section{Calculation of the fast equilibria}

We notice that $n(t)$ and $E(t)$, the global variables, are constants of motion of the fast process: migration and price variation. Fast equilibria are the solutions of the following system:

$$
\begin{aligned}
\sum_{i=1}^{L} m_{s i} n_{i}-\sum_{i=1}^{L} m_{i s} n_{s} & =0 \\
m_{i s} n_{s}-m_{s i} n_{i} & =0 \\
\beta_{i, i-1} E_{i-1}+\beta_{i, i+1} E_{i+1}-\left(\beta_{i-1, i}+\beta_{i+1, i}\right) E_{i} & =0 \\
D(p)-q \sum_{i=1}^{L} n_{i}^{*} E_{i}^{*} & =0
\end{aligned}
$$

Since $m_{i s}=\frac{m_{0}}{k_{s}}$ and $m_{s i}=\frac{m_{0}}{k_{i}}$ substituting $m_{i s}$ and $m_{s i}$ into the equation (1.2) leads to

$$
n_{i}^{*}=\frac{k_{i}}{k_{s}} n_{s}^{*}
$$

Since $n=\sum_{i=1}^{L} n_{i}^{*}+n_{s}^{*} \Rightarrow n=\sum_{i=1}^{L} \frac{k_{i}}{k_{s}} n_{s}^{*}+n_{s}^{*} \Rightarrow n=n_{s}^{*} \frac{\sum_{i=1}^{L} k_{i}+k_{s}}{k_{s}}$ leads to:

$$
n_{s}^{*}=\frac{k_{s}}{K} n \quad \text { and } n_{i}^{*}=\frac{k_{i}}{K} n
$$

Equation (1.3) states that $\beta_{1,2} E_{2}=\beta_{2,1} E_{1}$. As symmetric movement rates for boats were assumed, $\beta_{1,2}=\beta_{2,1}$ and therefore, $E_{1}=E_{2}$ at equilibrium. Then a simple recurrence shows that $\forall i, E_{i}=E_{i+1}$. As a consequence, we obtain $\forall i \in\{1, \ldots, L\} E_{i}^{*}=E_{1}^{*}$. So, with $E=\sum_{i=1}^{L} E_{i}^{*}$ we obtain the following 
result

$$
E_{i}^{*}=\frac{E}{L}
$$

From equation (1.4), we deduce that

$$
\begin{aligned}
D(p) & =q \sum_{i=1}^{L} \frac{k_{i}}{K} n \frac{E}{L} \\
& =q(1-\alpha) n \frac{E}{L} \\
& =Q n E
\end{aligned}
$$

where $Q=q(1-\alpha) / L$.

Because $D(p)=A-\gamma p$, at fast equilibrium, we have

$$
p^{*}=p^{*}(n, E)=\frac{A-Q n E}{\gamma}
$$

\section{Derivation of the aggregated model}

We substitute the fast equilibria computed in previous section and we obtain the following system:

$$
\begin{aligned}
\frac{d n_{s}}{d \tau} & =\varepsilon r_{s} n_{s}^{*}\left(1-\frac{n_{s}^{*}}{k_{s}}\right) \\
\frac{d n_{i}}{d \tau} & =\varepsilon\left(r_{1} n_{i}^{*}\left(1-\frac{n_{i}^{*}}{k_{i}}\right)-q n_{i}^{*} E_{i}^{*}\right) \\
\frac{d E_{i}}{d \tau} & =\varepsilon\left(-c+p^{*} q n_{i}^{*}\right) E_{i}^{*}
\end{aligned}
$$

Since $n=\sum_{i=1}^{L} n_{i}+n_{s}$ we have $\frac{d n}{d \tau}=\sum_{i=1}^{L} \frac{d n_{i}}{d \tau}+\frac{d n_{s}}{d \tau}$. We perform a change of timescale $t=\varepsilon \tau$. At slow time scale, the equation governing total fish stock reads:

$$
\begin{aligned}
\frac{d n}{d t} & =r_{s} n_{s}^{*}\left(1-\frac{n_{s}^{*}}{k_{s}}\right)+r_{1} \sum_{i=1}^{L} n_{i}^{*}\left(1-\frac{n_{i}^{*}}{k_{i}}\right)-q \sum_{i=1}^{L} n_{i}^{*} E_{i}^{*} \\
& =r_{s} \alpha n\left(1-\frac{n}{K}\right)+r_{1}(1-\alpha) n\left(1-\frac{n}{K}\right)-\frac{q(1-\alpha)}{L} n E \\
& =n\left(1-\frac{n}{K}\right)\left(\alpha r_{s}+(1-\alpha) r_{1}\right)-\frac{q(1-\alpha)}{L} n E
\end{aligned}
$$

We denote $r=\alpha r_{s}+(1-\alpha) r_{1}$ and $Q=\frac{q(1-\alpha)}{L}$. At slow time scale, the equation for fish stock finally reads:

$$
\frac{d n}{d t}=r n\left(1-\frac{n}{K}\right)-Q n E
$$

Equation governing total fishing efforts $E$ at slow time scale reads 


$$
\begin{aligned}
\frac{d E}{d t}=\sum_{i=1}^{L} \frac{d E_{i}}{d t} & =\sum_{i=1}^{L}\left(-c E_{i}^{*}+p^{*} q n_{i}^{*} E_{i}^{*}\right) \\
& =-c \sum_{i=1}^{L} E_{i}^{*}+p^{*} q \sum_{i=1}^{L} n_{i}^{*} E_{i}^{*} \\
& =\left(-c+p^{*} \frac{q(1-\alpha)}{L} n\right) E \\
& =\left(-c+p^{*} Q n\right) E
\end{aligned}
$$

At slow scale time we finally have

$$
\frac{d E}{d t}=\left(-c+p^{*} Q n\right) E
$$

where $p^{*}$ depends on $n$ and $E$ and is given by equation (1.9).

\section{Analysis of the non trivial equilibria}

System of equations (3.6) implies that fish stocks $\bar{n}$ at equilibria are roots of the following cubic equation:

$$
C(n)=\frac{r}{K} \bar{n}^{3}-r \bar{n}^{2}+A \bar{n}-\frac{\gamma c}{Q},
$$

i.e $C(\bar{n})=0$. First and second derivative of function $C$ read

$$
\begin{aligned}
C^{\prime}(n) & =\frac{3 r}{K} n^{2}-2 r n+A=\Psi(n) \\
C^{\prime \prime}(n) & =\frac{6 r}{K} n-2 r=\Psi^{\prime}(n)
\end{aligned}
$$

Notice that $C^{\prime}(\bar{n})$ and $\operatorname{det} J(\bar{n} ; \bar{E})$ have the same sign. $\Psi$ is a parabola and attains its minimum for $n=K / 3$. Its minimum value is $A-r K / 3$, so it is possible to distinguish two cases:

$-r<\frac{3 A}{K}: C^{\prime}$ is always positive. Function $C$ is increasing, and $C(K)=A K-\frac{\gamma c}{Q}$. Depending on the sign of $C(K)$, there are two cases:

(a) $\frac{A K Q}{\gamma}<c: c(K)<0$ and $c(\bar{n})=0$ implies that $n>K$. Then Equation (3.6) implies that $\bar{E}<0$. There is no positive equilibrium.

(b) $\frac{A K Q}{\gamma}>c: c(K)>0$ and $c(\bar{n})=0$ implies that $n<K$. There exists one positive equilibrium $(\bar{n} ; \bar{E}) . \Psi(\bar{n})>0$, so $\operatorname{det} J(\bar{n} ; \bar{E})>0$, and so $(\bar{n} ; \bar{E})$ is a stable equilibrium.

$-r>\frac{3 A}{K}: C^{\prime}\left(\right.$ or $\Psi$ ) has two roots $n_{1}$ and $n_{2}$ given by:

$$
0<n_{1}=\frac{K}{3}\left(1-\sqrt{1-\frac{3 A}{K r}}\right)<\frac{K}{3}<n_{2}=\frac{K}{3}\left(1+\sqrt{1-\frac{3 A}{K r}}\right)<K
$$

The following table of variation summarizes the possible cases: 


\begin{tabular}{|c|c|c|c|c|c|}
\hline$n$ & 0 & $n_{1}$ & & $n_{2}$ & \\
\hline$\Psi(n)$ & + & 0 & - & $\phi$ & + \\
\hline$C(n)$ & & & & & \\
\hline
\end{tabular}

Reminding that $\operatorname{det} J(\bar{n} ; \bar{E})$ and $\Psi(\bar{n})$ have the same sign, we distinguish three different subcases:

(a) $C\left(n_{1}\right)<0$ : there is a unique positive equilibrium $\left(\bar{n}_{3} ; \bar{E}_{3}\right)$. Because $\bar{n}_{3}>n_{2}, \Psi(\bar{n})_{3}>0$, and so it is stable.

(b) $C\left(n_{2}\right)>0$ : there is a unique positive equilibrium $\left(\bar{n}_{1} ; \bar{E}_{1}\right)$. Because $\bar{n}_{1}>n_{1}, \Psi(\bar{n})_{1}<0$, and so it is stable.

(c) $C\left(n_{1}\right)>0$ and $C\left(n_{2}\right)<0$ : there are three positive equilibrium points $\left(\bar{n}_{1} ; \bar{E}_{1}\right),\left(\bar{n}_{2} ; \bar{E}_{2}\right)$ and $\left(\bar{n}_{3} ; \bar{E}_{3}\right)$. They satisfying $\bar{n}_{1}<n_{1}<\bar{n}_{2}<n_{2}<\bar{n}_{3}$, so $\left(\bar{n}_{1} ; \bar{E}_{1}\right)$, and $\left(\bar{n}_{3} ; \bar{E}_{3}\right)$ are stable while $\left(\bar{n}_{2} ; \bar{E}_{2}\right)$ is a saddle.

\section{References}

[1] P. Auger, R. Mchich, N. Raïssi, B. Kooi. Effects of market price on the dynamics of a spatial fishery model: Overexploited fishery/traditional fishery. Ecol. Complex., 7 (2009), No. 1, 13 - 20. doi:10.1016/j.ecocom.2009.03.005

[2] P. Auger, J.C. Poggiale. Emergence of population growth models: fast migration and slow growth. J. Theor. Biol., 182 (1996), No. 2, 99-108. doi:10.1006/jtbi.1996.0145. PMID:8944142

[3] P. Auger, R. Bravo de la Parra, J.C. Poggiale, E. Sanchez, T. Nguyen-Huu. Aggregation of variables and applications to population dynamics. In Structured population models in biology and epidemiology. Lecture notes in mathematics. Vol. 1936. Edited by P. Magal and S. Ruan. Mathematical Biosciences Subseries (2008) Springer, Berlin., $209-263$.

[4] P. Auger, R. Roussarie. Complex ecological models with simple dynamics: from individuals to population. Acta Biotheor., 42 (1994), No. 2-3, 111-136. doi:10.1007/BF00709485.

[5] P. Auger, C. Lett, A. Moussaoui, S. Pioch. Optimal number of sites in artificial pelagic multi-site fisheries. Can. J. Fish. Aquat. Sci., 67 (2010), 296-303. doi:10.1139/F09-188

[6] E.B., Barbier, I. Strand, S. Sathirathai. Do open access conditions affect the valuation of an externality? Estimating the welfare effects of mangrove-fishery linkages. Env. Resour. Econ., 21 (2002), 343-367.

[7] C.W. Clark. Mathematical Bioeconomics: The Optimal Management of Renewable Resources. 2nd ed. Wiley, New York, 1990.

[8] L. Dagorn, K.N. Holland, D.G. Itano. Behavior of yellowfin (Thunnus albacares) and bigeye (T. obesus) tuna in a network of fish aggregating devices (FADs). Mar. Biol. (Berl.), 151(2) (2007) 595-606. doi:10.1007/s00227-006-0511-1.

[9] M. Robert, L. Dagorn, J. Lopez, G. Moreno, J.L.A. Deneubourg. Does social behavior influence the dynamics of aggregations formed by tropical tunas around floating objects? An experimental approach.. J. Exp. Mar. Biol. Ecol., 440 (2013), 238-243.

[10] M. de Lara, L. Doyen. Sustainable Management of renewable resources: Mathematical Models and Methods. SpringerVerlag, Berlin Heidelberg, 2008.

[11] Food and Agriculture Organization of the United Nations (FAO). Fishing Technology Equipments Fish Aggregating Device (FAD). Fisheries and Aquaculture Department.

[12] A. Fonteneau, J. Ariz, D. Gaertner, T. Nordstrom, P. Pallares. Observed changes in the species composition of tuna schools in the Gulf of Guinea between 1981 and 1999, in relation with the fish aggregrating device fishery. Aquat. Living Resour. 13 (2000) 253-257. doi:10.1016/S0990-7440(00)01054-8.

[13] C. Girard, S. Benhamou, L. Dagorn. FAD: fish aggregating device or fish attracting device? A new analysis of yellowfin tuna movements around floating objects. Anim. Behav. 67(2) (2004), 319-326. doi:10.1016/j.anbehav.2003.07.007.

[14] Y. Iwasa, V. Andreasen, S.A. Levin. Aggregation in model ecosystems. I. Perfect aggregation. Ecol. Model., 37 (1987), $287-302$.

[15] Y. Iwasa, S.A. Levin, V. Andreasen. Aggregation in model ecosystems. II. Approximate aggregation. IMA J. Math. Appl. Med. Biol., 6 (1989), 1-23.

[16] H. Kakimoto. Artificial fishing reef studies and effects. Japanese Institute of Technology on Fishing Ports, Grounds and Communities (JIFIC), 2, (2004), 150-178.

[17] L. Kumoro. Notes on the use of FADs in the Papua New Guinea purse seine fishery. Papua New Guinea National Fisheries Authority, Port Moresby, Papua New Guinea, 2003. 
[18] J.P. Kritzer, P.F. Sale. Metapopulation ecology in the sea: from Levins' model to marine ecology and fisheries science. Fish Fish. 5 (2004), 131-140.

[19] C.H. Lan, C.Y. Hsui. The deployment of artificial reef ecosystem: modelling, simulation and application. Simul. Model. Pract. Theory, 14 (2006), No. 5, 663-675. doi:10.1016/j.simpat.2005.10. 011.

[20] J.T. Lafrance. Linear demand functions in theory and practice. J. Econ. Theory, 37 (1985), 147-166.

[21] S.A. Levin, S. Pacala. Theories of simplification and scaling of spatially distributed processes, in: D. Tilman, P. Kareiva (Eds.), Spatial Ecology: The Role of Space in Population Dynamics and Interspecific Interactions, Princeton University, 1997.

[22] G. Moreno, L. Dagorn, G. Sancho, D. Itano. Fish behaviour from fishers' knowledge: the case study of tropical tuna around drifting fish aggregating devices (DFADs). Can. J. Fish. Aquat. Sci. 64(11) (2007) 1517-1528. doi:10.1139/F07113.

[23] I., Ohta, S. Kakuma. Periodic behavior and residence time of yellowfin and bigeye tuna associated with fish aggregating devices around Okinawa Islands, as identified with automated listening stations. Mar. Biol. (Berl.), 146(3) (2005), 581594. doi:10.1007/s00227-004-1456-x.

[24] J.N. Sanchirico, J.E. Wilen. Bioeconomics of spatial exploitation in a patchy environment . J. Environ. Econ. Manag. 37 (1999), 129-150.

[25] M.B. Schaefer. Some considerations of population dynamics and economics in relation to the management of the commercial marine fisheries. J. Fish. Res. Board Canada 14 (1957), 669-681.

[26] V.L. Smith. Economics of production from natural resources. Am. Econ. Rev., 58 (1968), No. 3, $409-431$.

[27] V.L. Smith. On models of commercial fishing. J. Political Economy, 77 (1969), No. 2, 181-198.

[28] L. Walras. Elément d'économie Politique Pure. Corbaz, Lausanne, 1874.

[29] Western and Central Pacific Fisheries Commission (WCPFC). Paragraph 24 of CMM 2008-01 FAD management and monitoring. Fifth regular session, Technical and Compliance Committee. WCPFC-TCC5-2009/22 (2009). 